\title{
Correction to: KGTK: A Toolkit for Large Knowledge Graph Manipulation and Analysis
}

\author{
Filip Ilievski (D), Daniel Garijo (D), Hans Chalupsky (D), \\ Naren Teja Divvala, Yixiang Yao (D), Craig Rogers (D), \\ Rongpeng Li (D), Jun Liu, Amandeep Singh (D), Daniel Schwabe (i), \\ and Pedro Szekely
}

\section{Correction to:}

Chapter "KGTK: A Toolkit for Large Knowledge Graph

Manipulation and Analysis" in: J. Z. Pan et al. (Eds.):

The Semantic Web - ISWC 2020, LNCS 12507,

https://doi.org/10.1007/978-3-030-62466-8_18

In the originally published version of chapter 18 the name of Rongpeng $\mathrm{Li}$ was misspelled. This has been corrected. 